\title{
Renal function associates with energy intake in elderly community-dwelling men
}

\author{
Desiree Luis ${ }^{1}$, Xiaoyan Huang ${ }^{1,2}$, Per Sjögren ${ }^{3}$, Ulf Risérus ${ }^{3}$, Johan Ärnlöv ${ }^{4,5}$, Bengt Lindholm ${ }^{1}$, \\ Tommy Cederholm ${ }^{3}$ and Juan Jesus Carrero ${ }^{1,6_{*}}$ \\ ${ }^{1}$ Divisions of Renal Medicine and Baxter Novum, Department of Clinical Science, Intervention, and Technology, \\ Karolinska Institutet, K56, Karolinska University Hospital Huddinge, S-141 86 Stockbolm, Sweden \\ ${ }^{2}$ Division of Nephrology, Peking University Shenzhen Hospital, Shenzhen, People's Republic of China \\ ${ }^{3}$ Department of Public Health and Caring Sciences, Clinical Nutrition and Metabolism, Uppsala University, Uppsala, Sweden \\ ${ }^{4}$ Department of Public Health and Caring Sciences, Section of Geriatrics, Uppsala University, Uppsala, Sweden \\ ${ }^{5}$ School of Health and Social Studies, Dalarna University, Falun, Sweden \\ ${ }^{6}$ Center for Molecular Medicine, Karolinska Institutet, Stockbolm, Sweden
}

(Submitted 28 August 2013 - Final revision received 9 January 2014 - Accepted 5 February 2014 - First published online 17 March 2014)

\begin{abstract}
Energy intake and renal function decrease with age. In patients with chronic kidney disease (CKD), spontaneous food intake decreases in parallel with the loss of renal function. The objective of the present study was to evaluate a possible relationship between renal dysfunction and energy intake in elderly community-dwelling men. A cross-sectional study including 1087 men aged 70 years from the Uppsala Longitudinal Study of Adult Men (ULSAM) community-based cohort was carried out. Dietary intake was assessed using $7 \mathrm{~d}$ food records, and glomerular filtration rate was estimated from serum cystatin C concentrations. Energy intake was normalised by ideal body weight, and macronutrient intake was energy-adjusted. The median normalised daily energy intake was 105 (interquartile range 88-124) kJ, and directly correlated with estimated glomerular filtration rate (eGFR) as determined by univariate analysis. Across the decreasing quartiles of eGFR, a significant trend of decreasing normalised energy intake was observed $(P=0 \cdot 01)$. A multivariable regression model including lifestyle factors and co-morbidities was used for predicting total energy intake. In this model, regular physical activity (standardised $\beta=0.160 ; P=0.008$ ), smoking (standardised $\beta=-0.081 ; P=0.008$ ), hypertension (standardised $\beta=-0.097 ; P=0.002$ ), hyperlipidaemia (standardised $\beta=-0.064 ; P=0.037$ ) and eGFR (per SD increase, standardised $\beta=0.064 ; P=0.04$ ) were found to be independent predictors of energy intake. Individuals with manifest CKD (eGFR $<60 \mathrm{ml} / \mathrm{min}$ per $1.73 \mathrm{~m}^{2}$ ) were more likely to have lower energy intake than those without. In conclusion, there was a direct and independent correlation between renal function and energy intake in a population-based cohort of elderly men. We speculate on a possible link between renal dysfunction and malnutrition in the elderly.
\end{abstract}

Key words: Nutrition: Chronic kidney disease: Elderly men: Dietary records

Malnutrition is an important factor that has been implicated in morbidity, mortality and impaired quality of life in the elderly $^{(1)}$. Both food intake and energy intake decrease with ageing $^{(2)}$, and many reasons including socio-economic conditions, physical and psychological disabilities, digestive troubles, difficulties with smelling, tasting or chewing and chronic diseases have been proposed to explain this phenomenon $^{(1)}$. The requirement for energy intake declines because of lower physical activity and lower $\mathrm{RMR}^{(3)}$, and altogether this may lead to deficiencies of essential nutrients ${ }^{(4)}$.
Gradual deterioration of kidney function is another feature of ageing. In national screening programmes, more than $50 \%$ of community-dwelling individuals above 65 years of age have been found to have some degree of chronic renal insufficiency that remains undiagnosed in the majority of cases ${ }^{(5)}$. It is well established that patients with chronic kidney disease (CKD) have a spontaneous reduction in food intake that increases in parallel with the progression of the disease (e.g. renal function

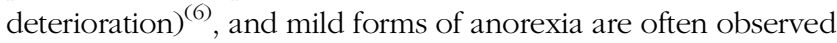
in patients with advanced $\mathrm{CKD}^{(7)}$. Multiple pathophysiological

Abbreviations: CKD, chronic kidney disease; eGFR, estimated glomerular filtration rate; ICD, International Classification of Diseases; UAER, urinary albumin excretion rate; ULSAM, Uppsala Longitudinal Study of Adult Men.

*Corresponding author: J. J. Carrero, email juan.jesus.carrero@ki.se 
pathways, such as retention of anorexigenic mediators, chronic inflammation, suppressed parasympathetic activity and yet uncharacterised uraemic toxicity, that originate from loss of renal function have been implicated in this process ${ }^{(8)}$. It is therefore possible that, at a general population level, the deterioration of renal function that occurs with ageing is yet another cause of reduced energy intake in the elderly. Therefore, the objective of the present study was to investigate the association between renal function and energy intake in a population-based community of elderly men.

\section{Materials and methods}

\section{Study population}

The present study was performed in the Uppsala Longitudinal Study of Adult Men (ULSAM) community-based cohort (described in detail at http://www2.pubcare.uu.se/ULSAM/), which is a population-based survey of all adult men aged 50 years in the Uppsala region with subsequent re-examinations. The present study was based on the third examination cycle of the ULSAM community-based cohort (during 1991 to 1995), when the attending participants were approximately 70 years of age and dietary records were collected for the first time. On this occasion, $73 \%$ of the eligible men (all Caucasians, $n$ 1221) agreed to participate. Exclusion criteria for this specific analysis were unavailability of data on $7 \mathrm{~d}$ dietary records and/or serum cystatin C concentrations ( $n$ 106), extreme values of reported energy intake $(<3200$ or $>18000 \mathrm{~kJ} / \mathrm{d}, n 5)$ and extreme values of energy intake per $\mathrm{kg}$ ideal body weight ( $>167 \mathrm{~kJ} / \mathrm{kg}$ per $\mathrm{d}, n$ 23). Therefore, the present analysis comprised 1087 men. All participants gave informed consent to participate. The study was approved by the ethics committee at Uppsala University.

\section{Clinical examinations}

All examinations, including anthropometric measurements, blood pressure, blood sampling, and questionnaires regarding medical history, smoking habits, education and physical activity level, were made under standardised conditions as described elsewhere $^{(9)}$. BMI was calculated as the ratio of body weight (in $\mathrm{kg}$ ) to height (in $\mathrm{m}^{2}$ ). The ideal body weight (IBW) was calculated by the Lorentz formula:

$$
\text { IBW men }=(\text { height }-100)-((\text { height }-150) / 4) .
$$

Smoking status was defined as current smoking $v$. non-smoking. Regular physical activity was defined according to four physical activity categories (sedentary, moderate, regular and athletic). Educational level was recorded as very low (elementary school), low (folk high school), medium (upper secondary school (6th form) or equivalent) and high (university or equivalent). Previous CVD was defined as history of any CVD as recorded in the Swedish Hospital Discharge Registry (International Classification of Diseases (ICD)-8 codes 390-458 or ICD-9 codes 390-459). Supine systolic and diastolic blood pressure levels were measured twice in the right arm after a $10 \mathrm{~min}$ rest, and means were calculated. Hypertension was defined as systolic blood pressure $\geq 140 \mathrm{mmHg}$, diastolic blood pressure $\geq 90 \mathrm{mmHg}$ or use of anti-hypertensive medications. Hyperlipidaemia was defined as serum cholesterol $>6.45 \mathrm{mmol} / 1$, serum TAG $>2.26 \mathrm{mmol} / 1$ and/or treatment with lipid-lowering medications. Diabetes mellitus was defined as fasting plasma glucose $\geq 7 \mathrm{mmol} / 1,2 \mathrm{~h}$ post-load glucose levels $\geq 11.1 \mathrm{mmol} / 1$ or the use of oral hypoglycaemic agents or insulin.

\section{Laboratory analysis}

Assays were performed at the Department of Clinical Chemistry, University Hospital, Uppsala, which is accredited according to the Swedish Board for Accreditation and Conformity Assessment (Swedac) standard ISO/IEC 17025 Venous blood samples were drawn after an overnight fast and stored at $-70^{\circ} \mathrm{C}$ until analyses. Serum TAG, total cholesterol and HDL cholesterol concentrations were assayed by enzymatic techniques. Serum albumin concentration was measured with spectrophotometry using bromine cresol green and reagents from Boehringer Mannheim. The instrument used was Hitachi 717 or 911 (Hitachi). The imprecision of the method was $3 \cdot 1 \%$ at the $33 \mathrm{~g} / 1$ level. Fasting blood glucose concentration was determined by an oxidase method. Urinary albumin excretion rate (UAER) was calculated from the amount of albumin in the urine collected during the night. All samples during the night and the first sample of morning urine were collected and used for the analysis. The assay used a commercially available RIA kit (Albumin RIA 100; Pharmacia). Serum cystatin C concentration was measured by latex-enhanced reagent ( $\mathrm{N}$ Latex Cystatin C; Dade Behring) with a Behring BN ProSpec analyser (Dade Behring). The total analytical imprecision of the method was $4.8 \%$ at $0.56 \mathrm{mg} / 1$ and $3.7 \%$ at $2.85 \mathrm{mg} / \mathrm{l}$. The estimated glomerular filtration rate (eGFR) was calculated from serum cystatin $\mathrm{C}$ concentrations (mg/l) by the following formula:

$$
y=77.24 \times x^{-1.2623}
$$

which has been shown to be closely correlated with iohexol clearance $^{(10)}$. Individuals with eGFR $<60 \mathrm{ml} / \mathrm{min}$ per $1.73 \mathrm{~m}^{2}$ were considered to have manifest CKD according to the current Kidney Disease Outcomes Quality Initiative definition.

\section{Dietary assessment}

Dietary habits were assessed using a $7 \mathrm{~d}$ dietary record based on a validated precoded menu book, which was prepared and previously used by the Swedish National Food Administration $^{(11)}$. A dietitian gave oral instructions to the participants on how to perform dietary registration, and the amounts consumed were reported in household measurements or specified as portion sizes. The daily intake of energy and various food items was calculated by using a database from the Swedish National Food Administration.

\section{Statistical analysis}

Statistical analyses were performed using statistical software SPSS version 21 (IBM SPSS Statistics). Results are expressed 
as medians and interquartile ranges or percentages of total. The eGFR was divided into quartiles. Spearman's univariate correlation coefficients $(\rho)$ were calculated to determine the correlations between eGFR and intake of energy and macronutrients. The Jonckheere-Terpstra test was used to assess trends across the quartiles of eGFR. Multivariable linear regression model was fitted to evaluate the association between eGFR and normalised energy intake by introducing potential confounders such as age, smoking status, physical activity, education, co-morbidities (CVD, hypertension, diabetes and hyperlipidaemia) and UAER. Data are expressed as standardised regression coefficients. This linear regression analysis was repeated using eGFR as the categorical variable $\left(<60 \mathrm{ml} / \mathrm{min}\right.$ per $1.73 \mathrm{~m}^{2} ; \geq 60 \mathrm{ml} / \mathrm{min}$ per $\left.1.73 \mathrm{~m}^{2}\right)$. $P$ values $<0.05$ were considered to be statistically significant.

\section{Results}

\section{General characteristics}

The median eGFR was 61.4 (interquartile range $52 \cdot 9-70 \cdot 1) \mathrm{ml} / \mathrm{min}$ per $1.73 \mathrm{~m}^{2}$, with values ranging from 10.5 to $109.2 \mathrm{ml} / \mathrm{min}$ per $1.73 \mathrm{~m}^{2}$. Participants were divided according to the quartiles of eGFR, and baseline characteristics are reported in Table 1 . Across the decreasing quartiles of eGFR, participants had a higher BMI, a higher prevalence of smokers, tended to be more sedentary and had a higher prevalence of hypertension and CVD. There was no difference regarding serum albumin, education, presence of diabetes and hyperlipidaemia.

\section{Dietary reports}

The median energy intake was 7226 (interquartile range 7226-8452) kJ. After normalisation by ideal body weight (Lorentz formula), the median energy intake was found to be 105 (interquartile range $88-124) \mathrm{kJ} / \mathrm{kg}$. The eGFR correlated directly, albeit weakly, with energy intake ( $\rho=0.09$; $P<0 \cdot 01$ ). Across the decreasing quartiles of eGFR (Table 2), a significant decreasing trend of energy intake was observed, but energy-adjusted macronutrient intake did not differ.

\section{Determinants of energy intake}

To provide further insight into the determinants of energy intake, a multivariable regression model including eGFR, age, lifestyle factors, co-morbidities as well as UAER was used (Table 3). In this model, regular physical activity, smoking, hypertension, hyperlipidaemia and eGFR were considered as independent predictors of energy intake. Using the quartiles of eGFR, individuals with manifest CKD (eGFR $<60 \mathrm{ml} / \mathrm{min}$ per $1.73 \mathrm{~m}^{2}$ ) were found to be associated with reduced energy intake in fully adjusted models. Subgroup analyses stratified by diabetic status or physical activity levels showed similar results (data not shown).

Table 1. Baseline characteristics according to the quartiles of estimated glomerular filtration rate (eGFR) in 1087 elderly men (Median values and interquartile ranges (IQR); number of subjects and percentages)

\begin{tabular}{|c|c|c|c|c|c|c|c|c|c|}
\hline \multirow{3}{*}{$\begin{array}{l}n . . . \\
\text { Parameters }\end{array}$} & \multicolumn{8}{|c|}{ Quartiles of eGFR (range, $\mathrm{ml} / \mathrm{min}$ per $1.73 \mathrm{~m}^{2}$ ) } & \multirow[b]{3}{*}{$P$ for trend ${ }^{\star}$} \\
\hline & \multicolumn{2}{|c|}{$\begin{array}{c}\text { Q1 }(111 \cdot 1-70 \cdot 2) \\
272\end{array}$} & \multicolumn{2}{|c|}{$\begin{array}{c}\text { Q2 (70.1-61.4) } \\
272\end{array}$} & \multicolumn{2}{|c|}{$\begin{array}{c}\text { Q3 (61.3-52.9) } \\
271\end{array}$} & \multicolumn{2}{|c|}{$\begin{array}{c}\text { Q4 (52.8-10.5) } \\
272\end{array}$} & \\
\hline & Median & IQR & Median & IQR & Median & IQR & Median & IQR & \\
\hline Age & 71 & $70 \cdot 4-71.4$ & 71 & $70 \cdot 6-71.4$ & 71 & $70.7-71.5$ & 71 & $70.7-71.5$ & 0.005 \\
\hline BMl $\left(\mathrm{kg} / \mathrm{m}^{2}\right)$ & $25 \cdot 6$ & $23 \cdot 7-27.9$ & $25 \cdot 7$ & $23 \cdot 9-27 \cdot 9$ & $25 \cdot 9$ & $23 \cdot 6-28 \cdot 3$ & $26 \cdot 4$ & $24 \cdot 2-28 \cdot 9$ & 0.014 \\
\hline Albumin $(g / l)$ & $42 \cdot 8$ & $41.2-44.5$ & 43.5 & $41 \cdot 7-45 \cdot 3$ & $43 \cdot 3$ & $41.4-44.9$ & $43 \cdot 1$ & $41.5-44.6$ & 0.604 \\
\hline \multirow[t]{2}{*}{ UAER (mg/24h) } & $7 \cdot 2$ & $4 \cdot 9-16$ & $6 \cdot 5$ & $4 \cdot 7-13 \cdot 7$ & $7 \cdot 6$ & $4 \cdot 8-15 \cdot 8$ & $7 \cdot 9$ & $4 \cdot 9-24$ & 0.117 \\
\hline & $n$ & $\%$ & $n$ & $\%$ & $n$ & $\%$ & $n$ & $\%$ & \\
\hline Smokers & 35 & $13 \cdot 5$ & 54 & $18 \cdot 8$ & 58 & 22.5 & 63 & $23 \cdot 2$ & 0.003 \\
\hline Physical activity & & & & & & & & & 0.015 \\
\hline Sedentary & 11 & $4 \cdot 3$ & 7 & $2 \cdot 5$ & 6 & $2 \cdot 4$ & 17 & $6 \cdot 3$ & \\
\hline Moderate & 76 & $29 \cdot 6$ & 98 & 35.6 & 90 & $36 \cdot 1$ & 99 & $36 \cdot 7$ & \\
\hline Regular & 151 & $58 \cdot 8$ & 151 & 54.9 & 141 & $56 \cdot 6$ & 142 & $52 \cdot 6$ & \\
\hline Athletic & 19 & $7 \cdot 4$ & 19 & $6 \cdot 9$ & 12 & 4.8 & 12 & 4.4 & \\
\hline Education & & & & & & & & & 0.887 \\
\hline Elementary school & 157 & $60 \cdot 2$ & 163 & $58 \cdot 4$ & 150 & $60 \cdot 2$ & 157 & 57.9 & \\
\hline Folk high school & 38 & $14 \cdot 6$ & 34 & 11.8 & 33 & $13 \cdot 3$ & 50 & 18.5 & \\
\hline Upper secondary school & 20 & $7 \cdot 7$ & 30 & $10 \cdot 5$ & 21 & 8.4 & 28 & $10 \cdot 3$ & \\
\hline University or equivalent & 46 & $17 \cdot 6$ & 52 & $18 \cdot 1$ & 45 & $18 \cdot 1$ & 36 & $13 \cdot 3$ & \\
\hline CVD & 65 & 24.4 & 71 & $24 \cdot 7$ & 88 & $33 \cdot 7$ & 109 & $39 \cdot 4$ & $<0.001$ \\
\hline Diabetes & 46 & $17 \cdot 3$ & 33 & 11.5 & 37 & $14 \cdot 2$ & 34 & $12 \cdot 3$ & 0.193 \\
\hline Hypertension & 64 & $24 \cdot 1$ & 79 & 27.5 & 87 & 33.3 & 115 & 41.5 & $<0.001$ \\
\hline Hyperlipidaemia & 97 & $36 \cdot 5$ & 93 & $32 \cdot 4$ & 101 & $38 \cdot 7$ & 95 & $34 \cdot 3$ & 0.990 \\
\hline
\end{tabular}

$Q$, quartile; UAER, urinary albumin excretion rate.

${ }^{*} P$ for trend determined using the Jonckheere-Terpstra test. 


\section{Discussion}

In the present analysis of the association between energy intake and renal function in a large population-based cohort of elderly men, there was a direct and independent correlation between energy intake and both eGFR and the presence of manifest CKD. This was observed over and above the expected contribution of co-morbidities, reduced physical activity or smoking.

Older adults generally have lower energy requirements than younger adults, mainly attributed to decreased physical activity, a lower RMR and age-associated changes in body composition characterised by a reduction in fat-free mass and muscle mass with an increase in visceral fat ${ }^{(12)}$. However, as many of these individuals do not reach the minimum necessary daily energy intake for adequate reserve of nutrients ${ }^{(13)}$, they become vulnerable to acute illnesses, chronic diseases and disability ${ }^{(14)}$. Indeed, malnutrition in the elderly is associated with an increased risk of both morbidity and mortality ${ }^{(15)}$.

We observed a median energy intake close to $7000 \mathrm{~kJ} / \mathrm{d}$ in the present cohort of elderly Swedish men. Although Lowenstein ${ }^{(13)}$ recommended $6276 \mathrm{~kJ} / \mathrm{d}(1500 \mathrm{kcal} / \mathrm{d})$ as the minimum requirement for maintaining adequate nutrition, subsequent studies have suggested that energy requirements may be higher in this population segment ${ }^{(14,16)}$. Many reasons have been proposed to explain the reduction in energy intake observed with ageing. Well-documented risk factors for poor nutrition include anorexia ${ }^{(17)}$, social isolation, depression ${ }^{(18)}$, dementia $^{(19)}$, eating disorders ${ }^{(20)}$, Parkinson's disease ${ }^{(21)}$, gastrointestinal dysfunction ${ }^{(22)}$ and chronic diseases such as hypertension $^{(23)}$, acute illness and polypharmacy ${ }^{(24)}$. Accordingly, in the present study, several of these co-morbidities and risk factors arise as independent contributors to the variations in energy intake. Low levels of physical activity in the elderly have also been found to be associated with poor energy intake and an increased risk of mortality and morbidity ${ }^{(25)}$. The present study is also in agreement with this finding, and complements those reports suggesting that increased leisure-time exercise and smoking cessation may improve energy intake, general health and survival in older men ${ }^{(26)}$.

Adding to this preceding evidence, the results of the present study suggest that renal function per se could additionally and independently contribute to explaining the variations in energy intake in the elderly. This observation extrapolates to the community the evidence available from previous studies in individuals with known CKD, suggesting that appetite and subsequent energy intake decreases in parallel with the reduction in renal function ${ }^{(27)}$. Anorexia is part of a complex geriatric syndrome common in older people ${ }^{(28)}$ that is associated with poor outcomes and several co-morbidities ${ }^{(29)}$. Similarly, patients with advanced CKD have often reported diminished or poor appetite, a condition associated with an increased risk of mortality and hospitalisation, and a significant worsening in their quality of life ${ }^{(7,8)}$. Plausible explanations for this phenomenon could be the role played by the kidney in the elimination of appetite suppressants and mediators, the development of anaemia, the retention and overproduction of inflammatory cytokines and adipokines and the suppression 
Table 3. Multivariable regression models predicting total energy intake, after normalisation by ideal body weight $(\mathrm{kJ} / \mathrm{kg}$ per $\mathrm{d})$

\begin{tabular}{lcc}
\hline & $\begin{array}{c}\text { Energy intake (per SD) } \\
\text { Standardised coefficients }\end{array}$ & $P$ \\
\hline Continuous model $^{*}$ & & \\
eGFR (per SD) & 0.064 & 0.047 \\
Regular physical activity & 0.141 & 0.012 \\
Smoking & -0.083 & 0.008 \\
Hypertension & -0.104 & 0.001 \\
Hyperlipidaemia & -0.063 & 0.045 \\
Dichotomous model ${ }^{*}+$ & & \\
eGFR $<60$ ml/min per $1.73 \mathrm{~m}^{2}$ & -0.066 & 0.038 \\
Regular physical activity & 0.145 & 0.009 \\
Smoking & -0.086 & 0.006 \\
Hypertension & -0.103 & 0.001 \\
Hyperlipidaemia & -0.062 & 0.049 \\
\hline
\end{tabular}

eGFR, estimated glomerular filtration rate.

* Models were adjusted for age, smoking status, physical activity, education, CVD, diabetes, hypertension, hyperlipidaemia and urinary albumin excretion rate.

†For kidney function, eGFR $>60 \mathrm{ml} / \mathrm{min}$ per $1.73 \mathrm{~m}^{2}$ was taken as a reference.

of parasympathetic activity, all of which can directly or indirectly influence the central regulation of feeding ${ }^{(8)}$. On the basis of this evidence, we speculate on a possible link between kidney dysfunction and malnutrition in the elderly. However, this needs to be formally demonstrated in populations with manifest malnutrition. Individuals included in the present study were indeed, in general, healthy, and their albumin levels did not differ across the strata of eGFR. Other measures of nutritional status such as recent body-weight loss or transferrin lacking in the present survey could have been adopted to explore this association. Lastly, given the present observational design, we cannot affirm that impaired renal function could be the cause of reduced energy intake. Conversely, it can be hypothesised that a chronic undernutrition state may contribute to renal disorders and organ failure. The role of nutrition in chronic disease certainly needs further exploration.

As a final remark, overweight and obesity are wellrecognised risk factors of mortality and the metabolic syndrome in the general population. They also represent a risk factor for progressive kidney damage ${ }^{(30)}$. In the present study, a positive trend towards increased BMI was observed across the individuals with reduced renal function, which may seem counter-intuitive given the fact that these individuals also presented with reduced energy intake. Excess fatness, however, does not exclude the presence of malnutrition, and it may represent the prelude to weight loss and underfeeding. Perhaps, as a reflection of this, some studies in elderly and CKD-specific populations have shown that mild obesity associates with lower mortality risk. The most likely explanation is that individuals with malnutrition/catabolism are at such a high risk that excess weight (wealth of energy) provides a measure of protection.

The large sample size and community representativeness are the strengths of the present study, together with the inclusion criteria that minimises confounding. Because of these inclusion criteria, the results of the present study apply to elderly men and are not necessarily extrapolable to elderly women. The use of $7 \mathrm{~d}$ food records is preferred over other dietary assessments in community studies, and also represents a strength of the present analysis. However, as with all dietary assessment tools, it is subjected to over- and under-reporting. Restricting inclusion of men of identical age provides a narrow range of renal function, which may underestimate the true strength of the associations observed. On the whole, we must acknowledge that the magnitude of the association reported in the present study is not very strong, and thus confirmation of the present hypothesis in independent community-dwelling cohorts with a broader renal function and range is necessary. Finally, although we carefully controlled for lifestyle parameters and concurrent co-morbidities, we acknowledge that these factors are intimately linked to the reduction of renal function per se, and it may not be easy to separate them from the consequences of ageing as such. We cannot exclude residual confounding by unmeasured factors such as the functional renal reserve, that is, the capacity of the kidney to augment its level of function above the baseline level when dietary demands are at place.

In conclusion, we report a direct correlation between renal function and energy intake in a population-based cohort of elderly men. This observation leads us to speculate that kidney dysfunction may be an additional cause of undernutrition in the elderly. Should this hypothesis be confirmed in independent community-dwelling cohorts, this vulnerable sector of the population may benefit from more stringent screening programmes and early identification (and management) of kidney damage.

\section{Acknowledgements}

D. L. was supported by a research exchange grant from the Spanish Society of Nephrology. J. J. C. acknowledges research grants from the Swedish Research Council, the Center for Gender Medicine and the Westman's Foundation.

D. L. carried out the studies and data analyses and drafted the manuscript. X. H. contributed with support on data analysis and interpretation. J. J. C. conceived the study, participated in its design and coordination and helped to draft the manuscript. The rest of the authors contributed to the development of the manuscript to its final form. P. S., U. R., J. A., B. L. and T. C. provided economic support as well as access to and management of the database. All authors read and approved the final version of the manuscript.

B. L. is employed by Baxter Healthcare Corporation, and Baxter Novum is the result of a grant from Baxter Healthcare Corporation to Karolinska Institutet. J. J. C. received speaker honoraria from Abbott Nutrition. The rest of the authors do not have any conflicts of interest to report.

\section{References}

1. Cope K (1996) Malnutrition in the elderly, a national crisis: contributes to disease, illness, disability, death, escalates health care costs, decreases quality of life. Seattle, WA: Region X, Administration on Aging. Publication No. 017-06200147-2. Washington DCUSG-mPO.

2. Kromhout D, de Lezenne Coulander C, Obermann-de Boer GL, et al. (1990) Changes in food and nutrient intake in middleaged men from 1960 to 1985 (the Zutphen Study). Am J Clin Nutr 51, 123-129. 
3. Visser M, Deurenberg P, van Staveren WA, et al. (1995) Resting metabolic rate and diet-induced thermogenesis in young and elderly subjects: relationship with body composition, fat distribution, and physical activity level. Am J Clin Nutr 61, 772-778.

4. Anonymous (1992) The nutrition of elderly people. Report of the Working Group on the Nutrition of Elderly People of the Committee on Medical Aspects of Food Policy. Rep Health Soc Subj (Lond) 43, 1-68.

5. James MT, Hemmelgarn BR \& Tonelli M (2010) Early recognition and prevention of chronic kidney disease. Lancet 375, 1296-1309.

6. Ikizler TA, Greene JH, Wingard RL, et al. (1995) Spontaneous dietary protein intake during progression of chronic renal failure. J Am Soc Nephrol 6, 1386-1391.

7. Carrero JJ, Qureshi AR, Axelsson J, et al. (2007) Comparison of nutritional and inflammatory markers in dialysis patients with reduced appetite. Am J Clin Nutr 85, 695-701.

8. Carrero JJ (2009) Identification of patients with eating disorders: clinical and biochemical signs of appetite loss in dialysis patients. J Ren Nutr 19, 10-15.

9. Vessby B, Tengblad S \& Lithell H (1994) Insulin sensitivity is related to the fatty acid composition of serum lipids and skeletal muscle phospholipids in 70-year-old men. Diabetologia 37, 1044-1050.

10. Larsson A, Malm J, Grubb A, et al. (2004) Calculation of glomerular filtration rate expressed in $\mathrm{mL} / \mathrm{min}$ from plasma cystatin C values in mg/L. Scand J Clin Lab Invest 64, 25-30.

11. Becker W (1994) [Food Habits and Intake in Sweden] (in Swedish). Uppsala, Sweden: The Swedish National Food Administration.

12. Henry CJ (2000) Mechanisms of changes in basal metabolism during ageing. Eur J Clin Nutr 54, Suppl. 3, S77-S91.

13. Lowenstein FW (1982) Nutritional status of the elderly in the United States of America, 1971-1974. J Am Coll Nutr 1, $165-177$.

14. de Groot CP, van den Broek T \& van Staveren W (1999) Energy intake and micronutrient intake in elderly Europeans: seeking the minimum requirement in the SENECA study. Age Ageing 28, 469-474.

15. Cornoni-Huntley JC, Harris TB, Everett DF, et al. (1991) An overview of body weight of older persons, including the impact on mortality. The National Health and Nutrition Examination Survey I - Epidemiologic Follow-up Study. J Clin Epidemiol 44, 743-753.
16. Black AE, Coward WA, Cole TJ, et al. (1996) Human energy expenditure in affluent societies: an analysis of 574 doublylabelled water measurements. Eur J Clin Nutr 50, 72-92.

17. Landi F, Liperoti R, Lattanzio F, et al. (2012) Effects of anorexia on mortality among older adults receiving home care: an observation study. J Nutr Health Aging 16, 79-83.

18. Saka B, Kaya O \& Ozturk GB (2010) Malnutrition in the elderly and its relationship with other geriatric syndromes. Clin Nutr 29, 745-748.

19. Stewart R, Masaki K, Xue QL, et al. (2005) A 32-year prospective study of change in body weight and incident dementia: the Honolulu-Asia Aging Study. Arch Neurol 62, 55-60.

20. Lapid MI, Prom MC, Burton MC, et al. (2010) Eating disorders in the elderly. Int Psychogeriatr 22, 523-536.

21. Sheard JM, Ash S, Mellick GD, et al. (2013) Markers of disease severity are associated with malnutrition in Parkinson's disease. PLOS ONE 8, e 57986.

22. Mamhidir AG, Ljunggren $G$, Kihlgren $M$, et al. (2006) Underweight, weight loss and related risk factors among older adults in sheltered housing - a Swedish follow-up study. J Nutr Health Aging 10, 255-262.

23. Guest JF, Panca M, Baeyens JP, et al. (2011) Health economic impact of managing patients following a community-based diagnosis of malnutrition in the UK. Clin Nutr 30, 422-429.

24. Visvanathan R, Macintosh C, Callary M, et al. (2003) The nutritional status of 250 older Australian recipients of domiciliary care services and its association with outcomes at 12 months. J Am Geriatr Soc 51, 1007-1011.

25. Fried LP \& Guralnik JM (1997) Disability in older adults: evidence regarding significance, etiology, and risk. J Am Geriatr Soc 45, 92-100.

26. Wannamethee SG, Shaper AG \& Walker M (1998) Changes in physical activity, mortality, and incidence of coronary heart disease in older men. Lancet 351, 1603-1608.

27. Fouque D, Vennegoor M, ter Wee P, et al. (2007) EBPG guideline on nutrition. Nephrol Dial Transplant 22, Suppl. 2, ii45-ii87.

28. Morley JE (1997) Anorexia of aging: physiologic and pathologic. Am J Clin Nutr 66, 760-773.

29. Landi F, Laviano A \& Cruz-Jentoft AJ (2010) The anorexia of aging: is it a geriatric syndrome? J Am Med Dir Assoc 11, 153-156.

30. Wang Y, Chen X \& Song Y (2008) Association between obesity and kidney disease: a systematic review and metaanalysis. Kidney Int 73, 19-33. 